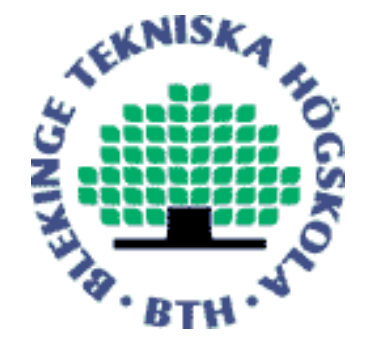

Copyright (C) 2010 IEEE.

Citation for the published paper:

Limit Cycles in an Industrially Applied Hybrid System

Anders Hultgren, Jan Melin, Per Ranstad

The 11th International Workshop on Variable structure systems

2010 Mexico city

This material is posted here with permission of the IEEE. Such permission of the IEEE does not in any way imply IEEE endorsement of any of BTH's products or services Internal or personal use of this material is permitted. However, permission to reprint/republish this material for advertising or promotional purposes or for creating new collective works for resale or redistribution must be obtained from the IEEE by sending a blank email message to pubs-permissions@iee.org.

By choosing to view this document, you agree to all provisions of the copyright laws protecting it. 


\title{
Limit Cycles in an Industrially Applied Hybrid System
}

\author{
Anders Hultgren*, Jan Melin** and Per Ranstad*** \\ *Blekinge Institute of Technology, School of Engineering, Sweden (Tel: +46 7094028 53, e-mail: anders.hultgren@bth.se) \\ **Linneaus University, School of Computer Science, Physics and Mathematics, Sweden (e-mail: jan.melin@lnu.se) \\ *** Alstom Power, PSP, Environmental R\&D, Sweden, (e-mail: per.ranstad@power.alstom.com)
}

\begin{abstract}
An industrially applied LCC power converter is modelled as a hybrid system. It is found that the hybrid system with three continuous states and one logic state given a parameter set up and different initial conditions has among other solutions one central closed trajectory and two types of limit cycles, on the left and the right side. The other solutions are indicated.
\end{abstract}

\section{INTRODUCTION}

DC/DC converters are today mainly controlled by switching devices causing abrupt changes in the converter dynamics. Modelling the switch devices as ideal, the controlled converters fall within the group of hybrid dynamical systems, see [1], [2] and [3]. The variety of topology of DC/DC converters is huge. In this report the control of an industrially applied resonant converter is discussed. Resonant converters have possibly many good properties, i.e. switching losses.

There are several suggestions of how to design a switched controller for resonant converters. Oruganti and Lee presented in [4] a set of switched state feedback controllers and since then several authors have addressed the design and analysis question of resonant converter controllers. See for instance [5], [6] and [7]. Some of the control laws suggested generate piecewise systems, possibly piecewise linear systems and some of others generate a more general subclass of hybrid systems.

Careful analysis of switched resonant converters, also when modelled with only two continuous states and as a piecewise linear system, can show that the possible solution set can be very rich. See for instance [8] and [9]. When modelling with three continuous states the solution set will increase even more, see [10]. In [11] a controller for an industrially applied resonant converter is analyzed. The converter is run by a trinary controller, i.e. the control output is a set with three values. In the current paper the same resonant converter is analysed running by a binary controller.

The industrially applied resonant converter, described in this report, is modeled as a series parallel, LCC, converter, see the circuit diagram in Fig. 1. The suggested controller is a switched feedback controller, derived to have high robustness for load disturbances. The solution set appears to be richer than expected and is together with the industrial implications addressed as the main topics in this report.
The series part of the converter consists of the capacitance, $\mathrm{C}$, and the inductance, $\mathrm{L}$. The parallel capacitance, $\mathrm{C}_{\mathrm{w}}$, models parasitic effects in the transformer, TR, and has the size of about $15 \%$ of the series capacitance, $\mathrm{C}$. The resistance $\mathrm{R}$ is neglected in the analysis. The controlled output of the converter is the current, $\mathrm{I}_{0}$, to the load capacitance, $\mathrm{C}_{0}$, on the secondary side of the transformer. The resonant converter is applied in a high voltage equipment. The capacitive load and high transformer ratio will give a comparatively large effective load capacitance, making the load voltage, $\mathrm{U}_{0}$, only slowly varying. Hence, the load voltage is modeled as a constant voltage source, with the value $\mathrm{U}_{0}$, , on the primary side of the transformer. The converter is supplied by the voltage, $\mathrm{E}$, and a full transistor bridge is controlling the voltage across the resonant circuit between junctions $\mathrm{A}$ and $\mathrm{B}, u_{A B}$. Assuming ideal transistor switches, the control signal can then be taken out of a set of two levels, $u_{A B} \in\{-E, E\}$, i.e. the controller is a binary controller. The resonance frequency is $20 \mathrm{kHz}$ and the controller is running the converter in a limit cycle above the resonance frequency, generating low switch-on losses in the transistors.

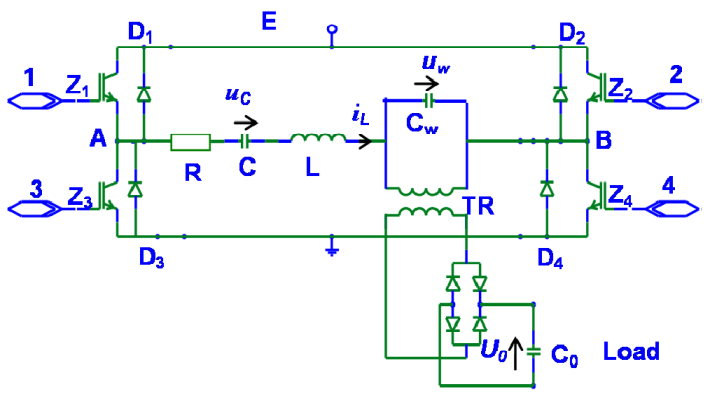

Fig. 1. The resonant converter used in an industrial application

In spite of the large load capacitance there are frequently load voltage disturbances in the industrial application that the controller has to cope with. The suggested controller is derived with the aim of robustness for load voltage disturbances. The controller is implemented in FPGA technology, implying that intensive calculations can be performed and that the sampling interval can be short. 


\section{THE CONVERTER MODEL}

\section{A. Choice of controller}

Choosing the two most important states as the normalized series capacitance voltage, $\mathrm{u}_{\mathrm{CN}}$, and inductance current, $\mathrm{i}_{\mathrm{LN}}$, which implies easier calculations, the resonant converter is given by the second order system

$$
\begin{aligned}
& \left(\begin{array}{l}
\dot{x} \\
\dot{y}
\end{array}\right)=\left(\begin{array}{cc}
0 & \frac{1}{\sqrt{L C}} \\
\frac{-1}{\sqrt{L C}} & 0
\end{array}\right)\left(\begin{array}{l}
x \\
y
\end{array}\right)+\left(\begin{array}{c}
0 \\
\frac{s-U_{0 N} \cdot \operatorname{sign}(y)}{\sqrt{L C}}
\end{array}\right) \\
& \text { where }\left(\begin{array}{ll}
\mathrm{x} & y
\end{array}\right)^{T}=\left(\begin{array}{ll}
u_{C N} & i_{L N}
\end{array}\right)^{T}=\left(\begin{array}{ll}
\frac{u_{C}}{E} & \frac{i_{L}}{E} \sqrt{\frac{L}{C}}
\end{array}\right)^{T} \\
& \text { and } U_{0 N}=\frac{U_{0}{ }^{\prime}}{E}, s=\frac{u_{A B}}{E} \in\{-1,1\}
\end{aligned}
$$

The system is run in a limit cycle by choosing $s=1$, each time the current $i_{L}$ change to positive sign and $s=-1$ each time current $i_{L}$ change to negative sign. By means of choosing $s=-$ $l$ at a certain state position $(x, y)$ on the upper half plane and $s$ $=1$ at a certain state position $(x, y)$ on the lower half plane the size of the limit cycle can be adjusted to stationary fit the chosen desired load current $I_{0 N}$ and the load voltage $U_{0 N}$. In Fig. 2 these switching positions on the upper half plane are shown as stars. The chosen control law for switching to $s=-l$ is then a switch curve that is the best ellipse that combine all stationary switch points for a certain load current and all different load voltages, see curves in Fig. 2. The ellipse radii are given as a function of the load current setpoint, $I_{O N}$, in TABLE I.

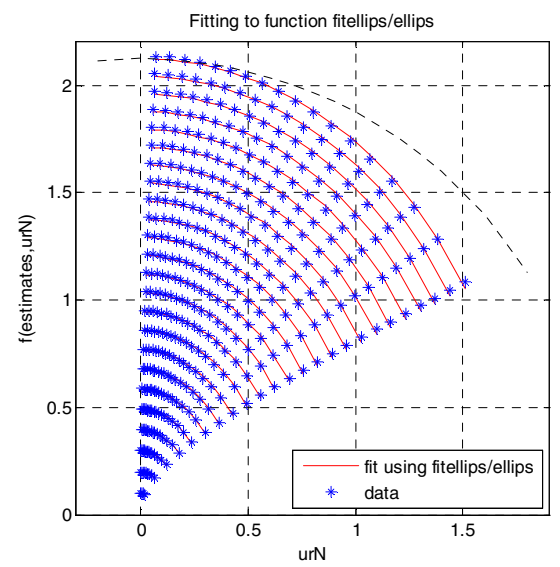

Fig. 2 Ellipses for different load current are shown in the upper half plane. The stars on a certain ellipse arc indicate the switch point (intersection point with the ellipse) for a certain load voltage.

\section{B. Modelling the closed third order system}

The chosen controller is evaluated for the third order system with the third state, $z$, as the normalized $u_{w}=u_{w} / E$. The closed system will be given by a hybrid dynamic model with three continuous states and two logic states. The three continuous states are given by the normalized capacitances voltages and the normalized inductance current,

Fig. 3. shows a state graph for the logic states given by the parameter $\mathrm{q}, q=1$ and $q=-1$, for the controlled system. The closed system is given by the system

$$
\left(\begin{array}{c}
\dot{x} \\
\dot{y} \\
\dot{z}
\end{array}\right)=\left(\begin{array}{ccc}
0 & \frac{1}{\sqrt{L C}} & 0 \\
\frac{-1}{\sqrt{L C}} & 0 & \frac{-1}{\sqrt{L C}} \\
0 & \Gamma & 0
\end{array}\right)\left(\begin{array}{l}
x \\
y \\
z
\end{array}\right)+\left(\begin{array}{c}
0 \\
\frac{1}{\sqrt{L C}} \\
0
\end{array}\right) \cdot \operatorname{sign}(y) \cdot q
$$

where

$$
\Gamma=\left\{\begin{array}{cl}
0, & \text { when } z \geq U_{0 N} \text { and } \mathrm{y}>0 \text { or } z \leq-U_{0 N} \text { and } \mathrm{y}<0 \\
\frac{\sqrt{C}}{C_{w} \sqrt{L}}, & \text { elsewhere }
\end{array}\right.
$$

and where $q$ is given by the state diagram in Fig. 3 .

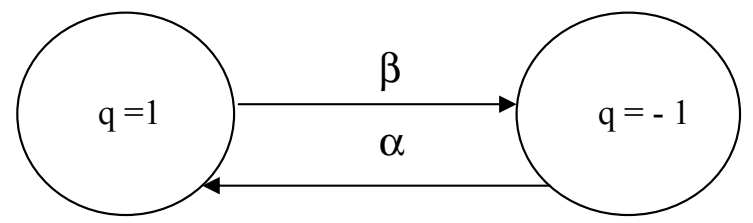

Fig. 3 State graph for the controlled converter

If we change the signs of the states, that is replace $x$ with $-x, y$ with $-y$ and $z$ with $-z$ and consider the property of $q$ we conclude that the system (2) is invariant and therefore the vector field of (2) has symmetric properties with respect to the origin. So called center symmetry.

The transition condition $a$, shown in Fig 3, is fulfilled if the state trajectory passes the $y=0$ plane and the transition condition $\beta$ is fulfilled if the state trajectory projected onto the xy-plane hits the chosen ellipse in the xy-plane. In the third order system, xyz, the elliptic switch curves will act as an elliptic cylinder. Fig. 7 shows the elliptic cylinder surface in the three dimensional system as indicated by three ellipses.

TABLE I

RADIE a IN $x$-DIRECTION AND b IN $y$-DIRECTION FOR THE DIFERENT ELLIPSES CORRESPONDING TO DIFFERENT LOAD CURRENTS, $\mathrm{I}_{0 \mathrm{~N}}$.

\begin{tabular}{|c|c|c|}
\hline $\mathrm{I} 0 \mathrm{~N}$ & Radie a (urN) & Radie b (irN) \\
\hline 0.0500 & 0.0606 & 0.0999 \\
\hline 0.1000 & 0.1235 & 0.1991 \\
\hline 0.1500 & 0.1887 & 0.2972 \\
\hline 0.2000 & 0.2557 & 0.3939 \\
\hline 0.2500 & 0.3243 & 0.4890 \\
\hline 0.3000 & 0.3940 & 0.5827 \\
\hline 0.3500 & 0.4648 & 0.6750 \\
\hline 0.4000 & 0.5364 & 0.7659 \\
\hline 0.4500 & 0.6087 & 0.8557 \\
\hline
\end{tabular}




\begin{tabular}{|l|l|l|}
\hline 0.5000 & 0.6816 & 0.9445 \\
\hline 0.5500 & 0.7551 & 1.0323 \\
\hline 0.6000 & 0.8290 & 1.1193 \\
\hline 0.6500 & 0.9033 & 1.2056 \\
\hline 0.7000 & 0.9781 & 1.2912 \\
\hline 0.7500 & 1.0530 & 1.3762 \\
\hline 0.8000 & 1.1282 & 1.4608 \\
\hline 0.8500 & 1.2038 & 1.5449 \\
\hline 0.9000 & 1.2795 & 1.6286 \\
\hline 0.9500 & 1.3554 & 1.7119 \\
\hline 1.0000 & 1.4314 & 1.7949 \\
\hline
\end{tabular}

\section{Following trajectories of the system}

In order to easier follow trajectories in the system the over all valid equation (2) is reformulated for different regions of the state space.

It is clear by (2) that the trajectories of the system always reside in between or onto the planes $\mathrm{z}=U_{0 \mathrm{~N}}$ and $\mathrm{z}=-U_{0 \mathrm{~N}}$ making $|z| \leq U_{0 \mathrm{~N}}$. The system can, when the trajectory resides in between these planes, be expressed by the following threedimensional hybrid

system:

$$
\left(\begin{array}{c}
\dot{x} \\
\dot{y} \\
\dot{z}
\end{array}\right)=\left(\begin{array}{ccc}
0 & \frac{1}{\sqrt{L C}} & 0 \\
\frac{-1}{\sqrt{L C}} & 0 & \frac{-1}{\sqrt{L C}} \\
0 & \frac{\sqrt{C}}{C_{w} \sqrt{L}} & 0
\end{array}\right)\left(\begin{array}{l}
x \\
y \\
z
\end{array}\right)+\left(\begin{array}{c}
0 \\
\frac{s}{\sqrt{L C}} \\
0
\end{array}\right)
$$

where $s=\mathrm{u}_{\mathrm{AB}} / E$ is a switched parameter $s \in\{-1,1\}$, that is the normalized control signal.

When the trajectory resides onto the limiting planes $z=U_{0 \mathrm{~N}}$ and $\mathrm{z}=-U_{0 \mathrm{~N}}$ the system is given by a twodimensional

$\left(\begin{array}{l}\dot{x} \\ \dot{y}\end{array}\right)=\left(\begin{array}{cc}0 & \frac{1}{\sqrt{L C}} \\ \frac{-1}{\sqrt{L C}} & 0\end{array}\right)\left(\begin{array}{l}x \\ y\end{array}\right)+\left(\begin{array}{c}0 \\ \frac{s-U_{0 N} \cdot \operatorname{sign}(y)}{\sqrt{L C}}\end{array}\right)$ system:

Considering the case when the trajectory resides between the two limiting planes and combining the first and the third equation of the three-dimensional model (4) we have $z=\frac{C}{C_{w}}$. $x+D$, where $D$ is an integration constant. Putting this in the second equation we obtain the following two-dimensional system projected onto the $x y$-plane:

$\left(\begin{array}{l}\dot{x} \\ \dot{y}\end{array}\right)=\left(\begin{array}{cc}0 & \frac{1}{\sqrt{L C}} \\ 1+\frac{C}{C_{w}} & 0\end{array}\right) \cdot\left(\begin{array}{l}x \\ y\end{array}\right)+\left(\begin{array}{c}0 \\ \frac{s-D}{\sqrt{L C}}\end{array}\right)$.
A compact elliptic cylinder is defined by $\Omega: \frac{x^{2}}{a^{2}}+\frac{y^{2}}{b^{2}} \leq 1$, where the half axis $a$ and $b$ are defined in section 1 above and given in Table $1 . \Omega$ is divided into the two sub regions $\Omega+$ : $y>0$ and $\Omega-: y<0$. Whenever a trajectory moves from $\Omega+$ to $\Omega$ - then $s=-1$, from $\Omega$ - to $\Omega+$ then $s=1$. After impact with the ellipse $\frac{x^{2}}{a^{2}}+\frac{y^{2}}{b^{2}}=1$, above the plane $\mathrm{y}=0$, the control variable is chosen to $s=-1$. After impact with the ellipse $\frac{x^{2}}{a^{2}}+\frac{y^{2}}{b^{2}}=1$, below the plane $\mathrm{y}=0$, the control variable is chosen to $s=1$. The switching surfaces are the plane $y=0$, the elliptic cylinder surface, the top of the cylinder $z=U_{0 \mathrm{~N}}$ and the bottom of the cylinder $z=-U_{0 \mathrm{~N}}$. The fixed points are the straight line $x+z=s$ in the plane $y=0$ and the eigenvalues are $\mu=0$ and $\mu= \pm \frac{i}{L} \cdot\left(\frac{1}{C}+\frac{1}{C_{w}}\right)$.

Note that this kind of switched system containing logic states is quite different from systems defined as piecewise, for example the LCC - converter defined in Melin et al. (2008).

\section{THE SYSTEM TRAJECTORIES}

In this section some properties of the trajectories and possible limit cycles are derived.

Conjecture. A trajectory that starts in the cylinder stays in the cylinder.

Consider the top of the cylinder, $z=U_{0 N}$, in the upper half plane, $y>0$. Immediately after an intersection between a trajectory and the ellipse at a point $\left(x_{0}, y_{0}\right)$ where $-a<x_{0}<$ $a$ and $0<y_{0}<b$ we have $s=0$ and from equation (5) we obtain the system: $\left(\begin{array}{c}\dot{x} \\ \dot{y}\end{array}\right)=\left(\begin{array}{c}\frac{y}{\sqrt{L C}} \\ -\frac{x+U_{0 N}}{\sqrt{L C}}\end{array}\right)$. The slope of the tangent at the intersection point is $\left|\frac{d y}{d x}\right|=-\frac{x_{0}+U_{0 N}}{y_{0}}$. The slope of the tangent of the ellipse is $\left|\frac{d y}{d x}\right|=-\frac{b^{2}}{a^{2}} \cdot \frac{x_{0}}{y_{0}}$. A sufficient condition for the trajectory to stay in the cylinder is $\left|\left(\frac{d y}{d x}\right)_{\text {trajectory }}\right|>\left|\left(\frac{d y}{d x}\right)_{\text {ellipse }}\right|$, this leads to the inequality
$x_{0}<\frac{U_{0 N}}{\left(\frac{b}{a}\right)^{2}-1}$. For each $I_{0 N}=0.1,0.2, \ldots, 0.9$ and $U_{0 N}=0.1,0.2, \ldots, 0.9$ simulations of the limit cycle show that the inequality is satisfied. A corresponding investigation has been done for limit cycles intersecting the cylinder between the top and the bottom as well as at the bottom of the cylinder and the inequality is still satisfied. This affirms the conjecture.

The section will now continue by calculating trajectories and derive a lemma.

When a trajectory is located in the region $|z|<U_{0 N}$ between the top and the bottom of the cylinder, according to the first 
and third equation of the system a family of planes $z=\frac{C}{C_{w}}$. $x+D$ are generated where the trajectory is located. For each starting point of the trajectory the plane is unique and the constant $D$ can be calculated. Using the equation of a plane and projecting the system onto the $x y$-plane and the trajectory is given by the equation $\frac{d y}{d x}=-\frac{\left(1+\frac{C}{C_{w}}\right) \cdot x-s+D}{y}$ with the solution $\frac{y^{2}}{2}=-\left(1+\frac{C}{C_{w}}\right) \cdot \frac{x^{2}}{2}+(s-D) \cdot x+F . \quad$ The integration constant $F$ is depending on the initial value.

At the bottom $z=-U_{0 N}$ of the cylinder the projected equation becomes $\frac{d y}{d x}=-\frac{x-U_{0 N}-s}{y}$ with the solution $\frac{y^{2}}{2}=$ $-\frac{x^{2}}{2}+\left(U_{0 N}+s\right) \cdot x+F$.

The corresponding solution at the top $z=U_{0 N}$ becomes $\frac{y^{2}}{2}=-\frac{x^{2}}{2}+\left(s-U_{0 N}\right) \cdot x+F$.

Considering the region entirely inside the cylinder $\Omega$ and chosing a starting point $P_{0}:\left(x_{0}, 0, z_{0}\right)$, the trajectory follows the plane $z=\frac{C}{C_{w}} \cdot x+D$ where $D=z_{0}-\frac{C}{C_{w}} \cdot x_{0}$ and enters the region $\Omega$ - and $s=-1$. The solution is given by $y^{2}=$ $-\left(1+\frac{C}{C_{w}}\right) x^{2}-2(D+1) x+\left(1+\frac{C}{C_{w}}\right) x_{0}^{2}+2(D+1) x_{0}$.

The trajectory then intersects the switching surface $y=0$ at $x_{1}=-x_{0}-\frac{2(D+1)}{1+\frac{C}{C_{w}}}$. The trajectory enters $\Omega+$ and follows a new plane upwards. Considering $s=1$ and using the initial value $x=x_{1}$ when $y=0$, the solution is given by $y^{2}=$ $-\left(1+\frac{C}{C_{w}}\right) x^{2}-2(D-1) x+\left(1+\frac{C}{C_{w}}\right) x_{0}^{2}+2(D+3) x_{0}+$ $\frac{8(D+1)}{1+\frac{C}{C_{w}}}$. The trajectory returns to the switching surface $y=$ 0 at $x_{2}=x_{0}+\frac{4}{1+\frac{C}{C_{w}}}$ which implies $x_{2} \neq x_{0}$. The following Lemma is proved.

Lemma. The system possesses no limit cycles entirely inside the cylinder, without intersecting the cylinder hull.

\section{Simulations}

Simulations indicate that the system possesses three different limit cycles and closed trajectories in the compact cylinder $\Omega$. One of them is unstable and centrally located in the cylinder. The other two are asymptotically stable and located to the left respectively to the right, see Fig. 4 .

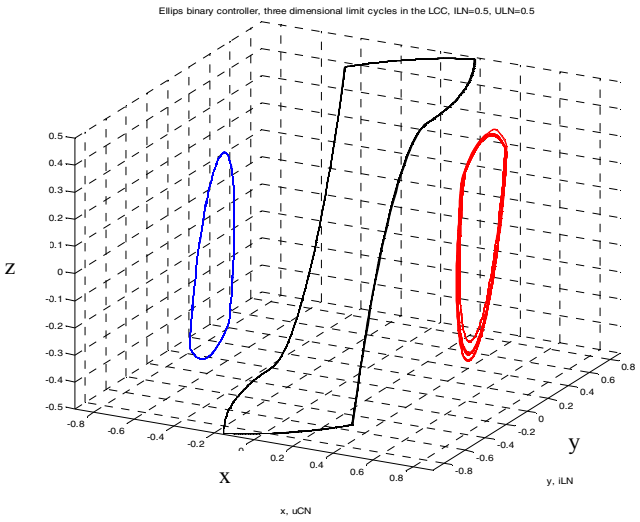

Fig. 4 Three different limit cycles and closed trajectories exist for the controlled converter, one unstable closed orbit, i.e. a closed trajectory in the central part and two stable limit cycles.

In Fig. 5 a xy projection of the limit cycles and closed trajectories are shown. One trajectory has initial values on the unstable closed trajectory. The other two have initial values close to them. It can be seen that one trajectory enters the left limit cycle and one the right limit cycle after some revolutions.

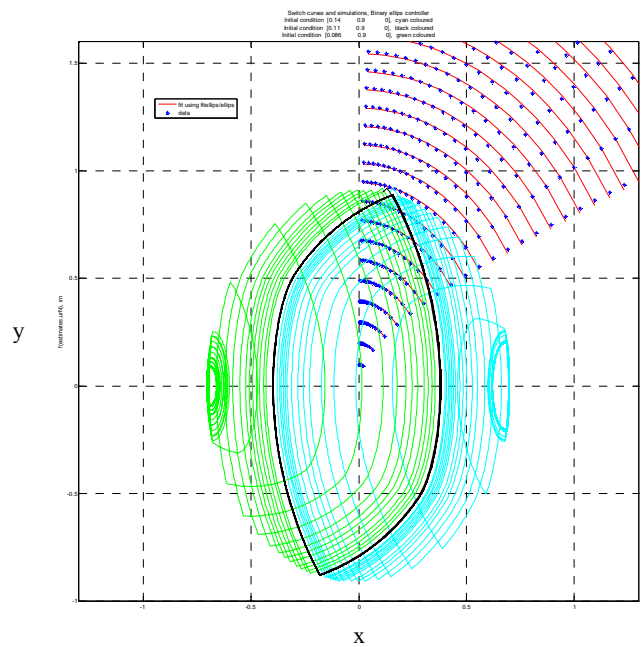

Fig. 5. The three limit cycles and closed trajectories shown in Fig. 4 are projected into the xy plane. A projection of part of the cylindric switch surface is also shown.

A detailed yz projection of the limit cycles and closed trajectories are given in Fig. 6 


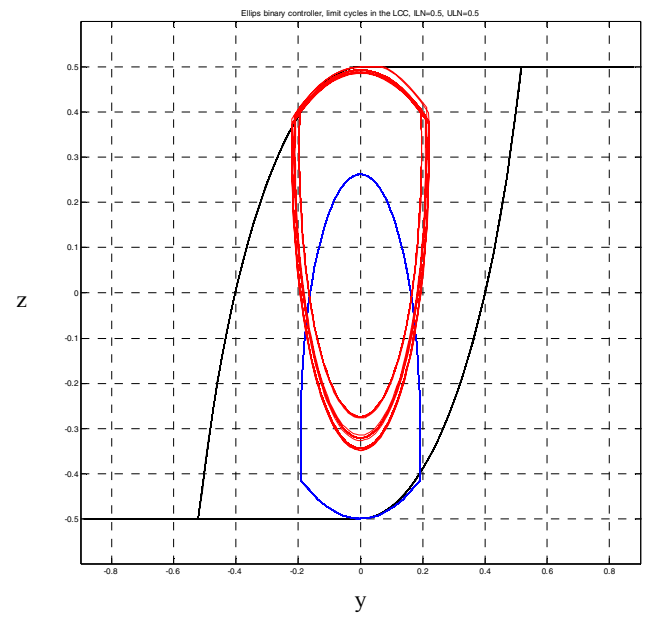

Fig. 6. The three limit cycles and closed trajectories shown in Fig. 4 are projected into the yz plane.

It can be seen that the limit cycle to the right is in fact a two revolutions limit cycle, see Fig. 7.

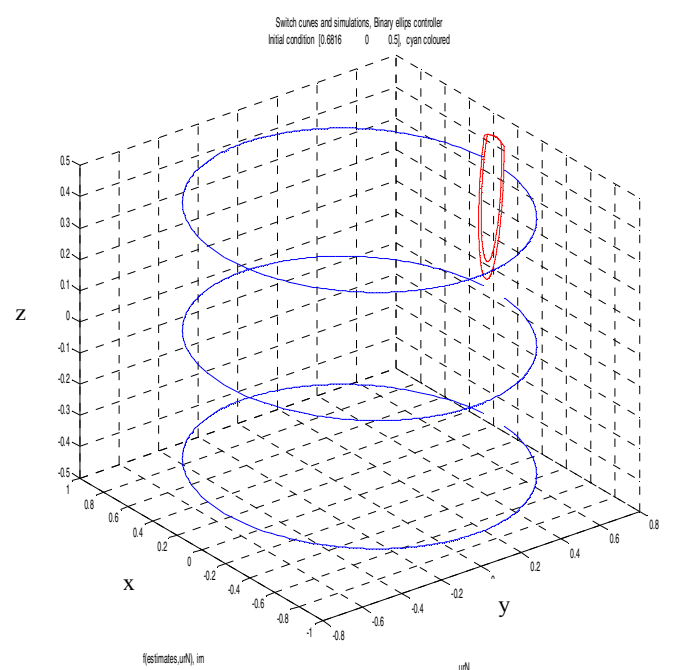

Fig. 7. One limit cycle to the right is shown together with an indication of the cylindric switch surface. It can be seen that the limit cycle consists of two revolutions.

In the figures Fig. 8 and Fig. 9 the limit cycles and closed trajectories can be seen for slightly different initial conditions.

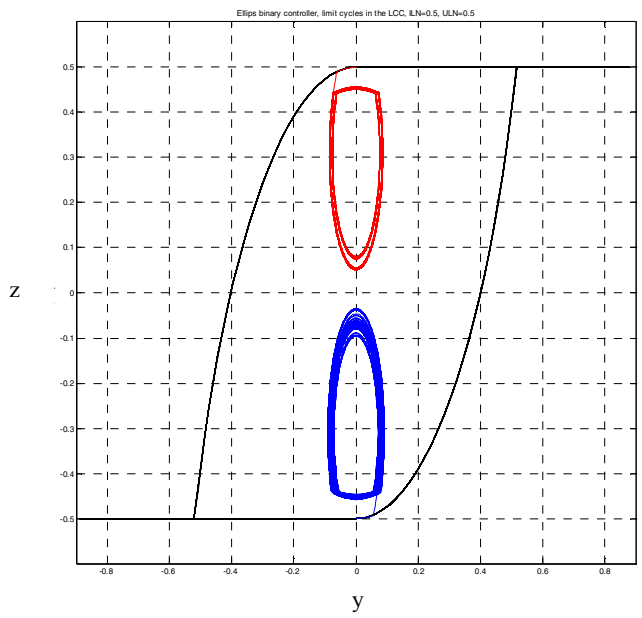

Fig. 8. Limit cycles and a closed trajectory. The two limit cycles have different initial conditions compared to the proceeding figures.

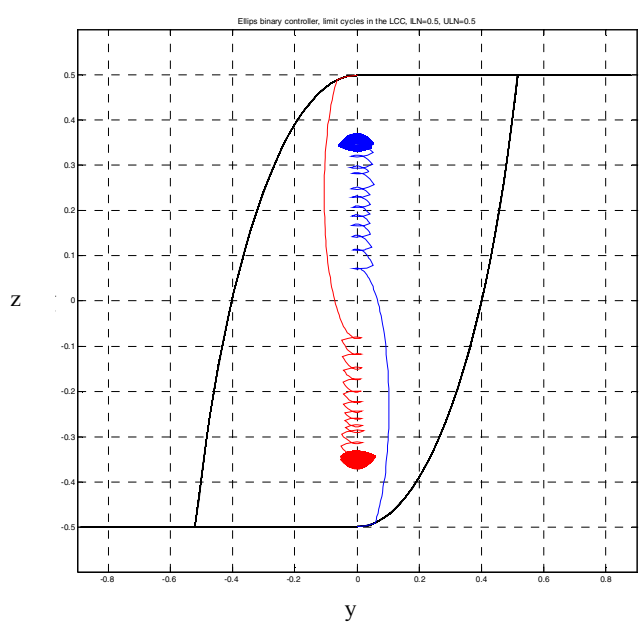

Fig. 9. A Closed trajectory and some trajectories for different initial conditions compared to the proceeding figures.

The limit cycles to left and to the right are situated very close to the cylinder boundary. It is possible that the limit cycle shown contains chattering phenomena.

\section{CONCLUSIONS}

An industrially applied LCC power converter is modelled as a hybrid system. It is found that the hybrid system given a parameter set up and different initial conditions has three different limit cycles. One central situated symmetrical closed trajectory and two types of asymmetric limit cycles, on the left 
and on the right. The two asymmetric limit cycles are stable while the third one is not.

It is judged that when introducing switched controllers in industrial applications the solution set has to be carefully analysed. The solution set of hybrid system can be very rich.

The aim in the industrial application is to deliver a certain rectified current to the load. This can be performed by letting a controller running the system in a stable limit cycle, symmetric around the origin. It has been found that the controller will only give a closed trajectory around the origin, i.e no stable limit cycle. Instead the suggested controller will give two highly asymmetric limit cycles. The asymmetric limit cycles will not generate the correct rectified current to the load.

\section{REFERENCES}

[1] Utkin V. Sliding modes in control optimization. Springer Verlag, 1992.

[2] Van der Schaft A. and Schumacher H. An introduction to Hybrid Dynamical Systems, Springer, 2000.

[3] Orlov Y.V. Discontinuous Systems. Springer 2009.

[4] Oruganti R. and Lee F.C. Resonant power Processors: Part 2-Methods of Control, IEEE-IAS ann. meet 1984.

[5] Rossetto L. A simple Control Technique for Series Resonant Converter. IEEE Transactions on Power Electronics, vol. 11, no. 4, pp 554-560, 1996.

[6] Chen H., Sng E., Tseng K.-J. Generalized Optimal Trajectory Control for Closed Loop Control of Series-Parallel Resonant Converter. IEEE Transactions on Power Electronics, vol. 21, no. 5, Sept. 2006.

[7] Hultgren A., Ranstad P., Lenells M. Ingelbrant P. and Nilsson M., "Switched Controllers Applied to a LCC Conv.", Proc. of NORpie/2002, IEEE and Pels, Stockholm, Sweden, Aug. 12-14 2002.

[8] Melin J. and Hultgren A. A limit cycle of a resonant converter. Proceedings of IFAC ADHS conference June 2003, St. Malo, France, 2003.

[9] Melin J. and Hultgren A. On conditions for regularity of solutions for a piecewise linear system. Journal of Nonlinear Analysis: Hybrid Systems and Applications, 65(12):2277-2301, 2006.

[10] Melin J., Hultgren A. and Lindström T. Two Types of Limit Cycles of a Resonant Converter Modelled by a Three-dimensional system. Journal of Nonlinear Analysis: Hybrid Systems and Applications, 2 (2008) 1275-1286 Elsevier Inc. 2008.

[11] Hultgren A., Melin J. and Ranstad P. Asymmetric Limit Cycles in an Industrially Applied Controlled Resonant Converter, $3^{\text {rd }}$ IFAC Conference on Analysis and Design of Hybrid systems, September 1618, 2009, Zaragoza, Spain. 